\title{
HYPER COMPLEX NUMBERS
}

\author{
* B. Gayathri Devi
}

\section{Introduction}

Hyper complex numbers were brought to our attention by Clyde Davenport, author of "A hyper complex calculus with application to relativity", ISBN 0-9623837-0-8. We shall start the introduction to hyper complex numbers by giving different types of definition given by different mathematician as follows:

- The hyper complex numbers are generalization of the complex numbers.

- Higher dimensional numbers are called hyper complex numbers, even though they do not share all the properties of complex numbers and no classical function theory can be constructed over them. (Clifford Algebraists)

- A hyper complex number is a number having properties departing from those of the real and complex numbers. (van der Warden (1985)

- Hyper complex numbers are extensions of the complex numbers such as quaternion, octonion, hexadecimalloniam etc.,

* Director of Studies (Maths), Department of Mathematics, Garden City College, Bangalore 560 049,vishwa_devi@hotmail.com. 
With this. few definition we can conclude that complex number can be viewed as a point in a plane, hyper complex numbers can be viewed as points in some higher dimensional Euclidean space; 4-dimension for quaternion, 8-dimesional for octonions and 16-dimensional for hexadecimallonions etc... The hyper complex numbers are a generalization of the complex numbers. They were created in an attempt to describe certain geometric operations in spaces with a dimension higher than 2-dimensional plane.

\section{History of Hyper Complex Numbers}

In 665 Brahmagupta (598-670) writes Khandakhadyaka that quadratic equation and allows for the possibility of negative solution. Nicholas Chuquet in 1484 introduced an exponenetial notation, allowing positive, negative and zero powers. In solving general equations he showed that some equation lead to imaginary solutions. Also Geronimo Cardano in 1545 used solutions to polynomials that lead to square roots of negative quantities, to get real solution by using a technique that later known as conjunction. (1572 Rafael Bombelli) John Wallis in 1673 publish his Algebra contains an early way to represent complex numbers geometrically. Euler showed the logarithm of a negative number is imaginary, also he published a paper in 1748 in which he mentioned the series formulations of $\exp (x), \sin (x)$ and $\cos (x)$, and deducing the formula exp $\left(i^{\circ}\right)=\cos (a)+i \sin (a)$, though this has discovered by Bernoulli in different forms. He also showed that complex numbers to the power complex number is also a complex numbers. D'Alemberts in 1749 function of complex variable is called Cauchy - Riemann equations. Euler made symbol i for root -1 in 1777. Jean Robert Argand in 1806 published the graphical representation of complex numbers, known a Argand plane was earlier discovered by Caspar Wessel (1745-1818) in 1797 as a two dimensional plane we unnoticed. Later on in 1814 Cauchy gave the first clear theory of functions of a complex variable. $\mathrm{He}$ showed that an analytic function of complex variable could be expanded about a point in a power series in the neighborhood of singularity. An Irish mathematician Rowan Hamiltonin first invented a number of non-commutative division algebra the year 1843. Quaternions are a single example of a more general class of hyper complex numbers.

\section{General form of Hyper Complex Numbers (Inductive Definition)}

It was wondered for some time whether this could be generalized to a 3-dimensional space i.e to number of the form a $+x i+y j$. It is known now that this is not possible. 
The only dimension in which there hypercomplex numbers which allow for a notion of division are dimension 4 or 8 and increase in dimension by multiple of 4 i.e $2^{n}$.

$$
a_{0}+a_{1} i_{1}+a_{2} i_{2}+\ldots .+a_{n} i_{n}
$$

where $\mathrm{n}$ is a fixed integer, and $a_{0}, a_{1} \ldots \ldots a_{n}$ are arbitrary real numbers and $i_{0}, i_{i} \ldots . . . . . i_{n}$ are symbols such that

$$
a_{0}+a_{1} i_{1}+a_{2} i_{2}+\ldots \ldots+a_{n} i_{n}=b_{0}+b_{1} i_{1}+b_{2} i_{2}+\ldots .+b_{n} i_{n}
$$

If and if only

$$
a_{0}=b_{0} \quad a_{1}=b_{1} \quad \ldots \ldots \ldots \ldots . . . \quad a_{n}=b_{n}
$$

Equation $I$ is said to be $n^{\text {th }}$ complex number. Each multiplication of 2 bases $i_{0}$ and $i_{b}$ is necessarily a member of the set of hyper complex number being defined. If given two integer from 1 to $n$, $a$ and $b$ and read numbers $p_{0}$ through $p_{n}$.

We can define a multiplication table such that,

$$
i_{0} i_{b}=p_{0}+p_{1} i_{1}+p_{2} i_{2}+\ldots \ldots+p_{n} i_{n}
$$

Therefore, for an $n$th order hyper complex number, $n^{*} n^{*}(n+1)$ number of such constant must be defined to determine the form of the algebra.
1. Ex: Read Numbers
Oth order
require none
2. Ex: Complex Numbers
1st order
require two
3. Ex: Quaternions
$3^{\text {rd }}$ order
require $3.3 .4=36$

Caley-Dickson constructed a procedure by which a $2^{n}$-dimensional hyper complex number is constructed from a pair of $2(n-1)$ dimensional hyper complex numbers where $n$ is a positive integer. This is accomplished by definining the multiplication rule for the two $2^{n}$-dimensional hyper complex numbers in terms of the $2(n-1)$ dimensional hyper complex numbers.

2-dimensional complex number $(n=1)$

4-dimensional complex number $(n=2)$ (Quaternions)

8-dimensional complex number $(n=3)$ (Octonions)

16-dimensional complex number $(n=4)$ (Hexadecimallonians) 
32-dimensional complex number $(n=5)$

64-dimensional complex number $(n=6)$

etc..., can all be constructed from real numbers by the iteration of this process. At each iteration some new basal elements, ik are introduced with the property $\mathrm{i}^{2}=-1$. In the following we will choose the (field parameter) ${ }^{*} \mu=-1$ and construct

1) Complex number from real numbers

2) Quaternion from complex numbers

3) Octonions from quaternion

\section{Field Parameter}

Let $X$ and $Y$ be two $2^{n}$-dimensional hyper complex numbers. Let $X, Y$ be ordered pair of $2(n-1)$-dimensional hyper complex numbers $A, B, C$ and $D$ such that $X=(A ; B)$, $Y=(C ; D)$ According to Caley-Dickson procedure of $X$ and $Y$ is defined as follows.

$$
\begin{aligned}
& X Y=(A ; B)(C ; D) \\
& =\left(A C+\mu D^{*} B ; B C+D A\right)
\end{aligned}
$$

where $D^{*}$ and $C^{*}$ are conjugate of $D$ and $C$ respectively and $\mu$ is field parameter.

Complex number $(n=1)$

Consider two complex numbers $Z_{1}=a+b i$ and $Z_{2}=c+$ di where $a, b, c$, d are elements of $R$ and $i^{2}=-1$

Then

$$
z_{1} z_{2}=(a c-b d)+(a d+b c) i_{1}
$$

When this is compared with Caley-Dickson multiplication rule for $Z_{1}=(a, b)$ and $Z_{2}=(c, d)$ with

$$
\mu=-1, Z_{1} Z_{2}=(a c-b d) ;(a d+b c),
$$




$$
\begin{aligned}
& d^{*}=d \text { d elements of } R \\
& c^{*}=c \quad c \text { elements of } R
\end{aligned}
$$

which clearly shows that $(a c-b d, 0)$ and $(0, a d+b c)$ correspond to $(a c-b d)$ and $(\mathrm{ad}+\mathrm{bc}) i_{1}$, respectively.

\section{Quaternian ( $n=2)$}

In order to construct a quaternian are used to introduce another imaginary unit $i_{2}$ with the property $i^{2}=-1$, but different from $i_{1}$. Write fwo quaternion $P \& Q$, each in terms of two complex numbers.

$$
P=A+B i_{2}, Q=C+D i_{2}
$$

where,

$$
\begin{aligned}
& \left(A+B i_{2}\right),\left(C+D i_{2}\right)=A C+B i_{2}+A D i_{2}+B i_{2} D_{2} \\
& =A C+B C^{*} i_{2}+D A i_{2}+B{ }^{*} i_{2} i_{2} \\
& =\left(A C-D^{*} B\right)+\left(B C^{*}+D A\right) i_{2}
\end{aligned}
$$

Compare this with the Caley-Dickson multiplication of two quaternions defined as ordered pair of Complex numbers,

$$
\begin{aligned}
& (A ; B) \text { and }(C ; D) \text {, with } \mu=-1 \\
& P Q=(A, B)(C, D)=\left(A C-D^{*} B\right) ;\left(B C^{*}+D A\right)
\end{aligned}
$$

Note that in the expression for $P=A+B i_{2}$, there in a term consisting of $i_{1}, i_{2}$. This represents a new imaginary number a basal element of quaternion $i_{3}=i_{1} i_{2}$.

\section{Octonions $(n=3)$}

Let $P, Q, R, S$ be four quaternion. Define two octonions, $M$ and $N$, as the ordered pair, $M=(P, Q), N=(R, S)$. Then the multiplication of $M$ and $N$ is given by

$$
M N=\left(P R+U S^{*} Q, Q R^{*}+S P\right)
$$

The new basal elements introduced in the process are $i_{4}, i_{5}, i_{6}$ and $i_{6}$. 


\section{Hexadecimalions $(n=4)$}

If we try to do same thing with hexadecimalions we find that neither $\left(1, e_{1}\right.$, $\left.e_{2} \ldots \ldots . e_{16}\right)$ nor $\left(e_{16}, e_{15} \ldots \ldots . e_{1}\right)$ are rotation in general even if $\|e\|=1$. That trails ends as well.

Note: if $i_{2}$ is another imaginary number with property $\left(i_{2}\right)=-1$ but different from $i_{1}$, such that

$$
\begin{aligned}
& i_{2} i_{1}=-i_{1} i_{2} \text { then } \\
& i_{2} z_{1}=i_{2}(a+b i) \\
& =a i_{2}+b i_{1} i_{2}=z^{*} i_{2}
\end{aligned}
$$

Above equation are said to be $n^{\text {th }}$ order complex numbers. Each multiplication of 2 bases $i_{a}$ and $i_{b}$ is necessarily a member of set of hyper complex numbers being defined. If given two integers from 1 to $n, a$ and $b$ and read numbers $p_{0}$ through $p_{n}$. We can define a multiplication table such that,

$$
i_{0} i_{b}=p_{0}+p_{1} i_{1}+p_{2} i_{2}+\ldots \ldots+p_{n} i_{n}
$$

Note: Operation on the complex numbers can be used to describe many of the geometric operation the plane. For instance multiplication by a real number corresponds to a scaling of the plane. Multiplication by complex numbers with a modulus (length) of 1 corresponds to a rotation of the plane. Adding complex numbers corresponds to the translation of the plane.

\section{Properties that all Hyper Complex Numbers obey}

However hyper complex numbers have a wonderful property for fractal purposes. Every function defined for complex numbers has a simple generalization to hyper complex number. Fractint's implementation takes advantage of this by using " $\mathrm{f}$ " variables -the iteration formula is:

$$
n(n+1)=f n(n(n))+c
$$

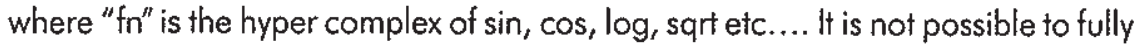
generalize the complex number to 4-dimension without sacrificing some of the algebraic properties shared by real and complex number. Quaternion violates the Commutative law of multiplication. Similarly octonions are non-associative. Hyper complex number fail the rule that says all non zero element have multiplicative 
inverse i.e. If $z$ is non-zero, there should be a number $1 / z$ such that $(1 / z) \times(z)=1$. This law holds most of the time but not all the time for hyper complex number.

\section{More about Quaternion and Octonion}

$$
\begin{aligned}
& \mathbf{i}=\left[\begin{array}{cc}
-\mathbf{i} & 0 \\
0 & -\mathbf{i}
\end{array}\right] \\
& \mathbf{j}=\left[\begin{array}{cc}
0 & 1 \\
-1 & 0
\end{array}\right] \\
& \mathbf{k}=\left[\begin{array}{ll}
0 & \mathbf{i} \\
\mathbf{i} & 0
\end{array}\right]
\end{aligned}
$$

$U$ is used to denote the identity matrix for $I$. Here it can be noticed that matrix are closely related to the Pauli spin matrices combined with the identity matrix.

$$
\begin{aligned}
& i^{2}=-u \\
& j^{2}=-v \\
& k^{2}=-w
\end{aligned}
$$

Therefore, $i, i, k$ are three essential different solution of the matrix equation.

$$
x^{2}=-4
$$

This could be considered the square roots of the negative identity matrix. A linear combination of basic quaternion with integer coefficients is sometime called a Hamilton integer. In R4 the basic of the quaternion can be given by 


$$
\begin{aligned}
& i=\left[\begin{array}{cccc}
0 & 1 & 0 & 0 \\
-1 & 0 & 0 & 0 \\
0 & 0 & 0 & 1 \\
0 & 0 & -1 & 0
\end{array}\right] \\
& j=\left[\begin{array}{cccc}
0 & 0 & 0 & -1 \\
0 & 0 & -1 & 0 \\
0 & 1 & 0 & 0 \\
1 & 0 & 0 & 0
\end{array}\right] \\
& \mathrm{k}=\left[\begin{array}{cccc}
0 & 0 & -1 & 0 \\
0 & 0 & 0 & 1 \\
1 & 0 & 0 & 0 \\
0 & 1 & 0 & 0 \\
0 & 0 & 0 & 0 \\
0 & -1 & 0 & 0 \\
0 & 0 & 0 & 1
\end{array}\right] \\
& 0
\end{aligned}
$$


The quaternion satisfies the following identities, sometimes known as Hamilton rules.

$$
\begin{aligned}
& \mathrm{i}^{2}=\mathrm{i}^{2}=\mathrm{k}^{2}=-1 \\
& \mathrm{ij}=-\mathrm{i}=\mathrm{k} \quad \mathrm{ik}=-\mathrm{ki}=\mathrm{i} \quad \mathrm{ki}=-\mathrm{ik}=\mathrm{i}
\end{aligned}
$$

They have the following multiplication table

\begin{tabular}{|c|c|c|c|c|}
\hline & 1 & $i$ & $i$ & $k$ \\
\hline 1 & 1 & $i$ & $i$ & $k$ \\
\hline$i$ & $i$ & $-k$ & -1 & $-i$ \\
\hline$i$ & $i$ & $-k$ & -1 & 1 \\
\hline$k$ & $k$ & $i$ & $-i$ & -1 \\
\hline
\end{tabular}

The quaternion $\pm 1, \pm i, \pm j$ and $\pm k$ form a non-abelian group of order eight with multiplication as the group operation known as $Q_{8}$ of $H$. The quaternion can be written in the form

$$
a=a_{1}+a_{2} i+a_{3} i+a_{4} k
$$

and its conjugate

$$
a=a_{1}-a_{2} i-a_{3} i-a_{4} k
$$

Sum of quaternion is

$$
a+b=\left(a_{1}+b_{1}\right)+\left(a_{2}+b_{2}\right) i+\left(a_{3}+b_{3}\right) i+\left(a_{4}+b_{4}\right) k
$$

Product of quaternion is

$$
\begin{aligned}
& a b=\left(a_{1} b_{1}-a_{2} b_{2}-a_{3} b_{3}-a_{4} b_{4}\right)+\left(a_{1} b_{2}+a_{2} b_{1}+a_{3} b_{4}-a_{4} b_{3}\right) i \\
& +\left(a_{1} b_{3}-a_{2} b_{4}+a_{3} b_{1}+a_{4} b_{2}\right) j+\left(a_{3} b_{4}+a_{2} b_{3}-a_{3} b_{2}+a_{4} b_{1}\right) k
\end{aligned}
$$

The quaternion norm therefore defined by

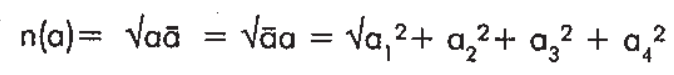

represent very close relationship with four vectors. Instead of having one real component and only one imaginary component, Quaternion has one real component and three imaginary components. Quaternion can be interpreted as a scalar plus and vector by writing as 
where

$$
(s, v)=s+v_{x} i+v_{y} i+v_{z} k
$$

$$
\begin{aligned}
& i^{2}=i^{2}=k^{2}=-1 \\
& i j=k, \quad j k=i, \quad k i=i
\end{aligned}
$$

and

$$
i i=-k, k j=-i, i k=-i
$$

Quaternion are also explained as the complex numbers which are useful to represent and to do operation on, points in 2-D space, quaternion are an extension of this into 3-D space.

If $(s, v)$ is quaternion

$$
(s, v)=s+v_{x} i+v_{y} j+v_{z} k=q
$$

Then

$$
q^{\prime}=\operatorname{conj}(q)=s-v_{x} i-v_{y} j-v_{z} k
$$

Magnitude $=\|q\|=\operatorname{sqr}\left(q^{*} q^{\prime}\right)=\operatorname{sqr}\left(a^{*} a=b^{*} b=c^{*} c=d^{*} d\right)$

Then the next question is why are 4 numbers used to represent 3-D space? The real part s represent the scalar value, and the imaginary part $\left(v_{x}, v_{y}, v_{z}\right)$ is a vector that defines the axis of rotation. If a quaternion $q$ has length 1 , we say $q$ is a unit quaternion. The inverse of a unit quaternion is its conjugate $=q^{\prime}$.

\section{Representing Rotation with quaternion}

To represent the angle tfrom the unit vector $u, q=\cos (t / 2)+u \sin (t / 2)$. After rotation, a point $p=\{0, p)$ is then given by $p^{\prime}=q p(q)=q p q^{\prime} n(q)=1$ be computed using the identity

$$
q_{2}\left(q_{1} p q_{1}^{\prime}\right) q_{2}^{\prime}=\left(q_{2} q_{1}\right) p\left(q_{1}^{\prime} q_{2}^{\prime}\right)=\left(q_{2} q_{1}\right) p q_{2}^{\prime} q_{1}^{\prime}
$$

\section{Concatenating Rotation}

To calculate the result of result of rotation represented by $q$ followed by $p$ we have to calculate the cross product $p^{*} q$ therefore when working with rotation we do not need to convert to matrices as we can with quaternion multiplication only. 


\section{Octonions}

Soon after the discovery of quaternion algebra, Graves an Cayley discovered octonions algebra independently, using eight object $e_{\mu}(\mu=0,1,2, \ldots \ldots . .7)$ instead of four, as of quaternion, while the multiplication rule for $e_{i}(j=1,2, \ldots \ldots \ldots, 7)$ is given by

1) $e_{i}^{2}=-e_{0}(j=1,2 \ldots \ldots \ldots 7)$

2) $e_{i} e_{k}=-e_{k} e_{i}(i, k=1,2 \ldots \ldots .7)$

3) The only non zero product involving different ej and ek ( $i \neq k)$ are

$$
\begin{array}{llll}
e_{1} e_{2}=e_{3^{\prime}} & e_{5} e_{1}=e_{6^{\prime}} & e_{6} e_{2}=e_{4^{\prime}} & e_{4} e_{3}=e_{5^{\prime}} \\
e_{4} e_{7}=-e_{1^{\prime}} & e_{6} e_{7}=-e_{3^{\prime}} & e_{5} e_{7}=-e_{2}
\end{array}
$$

and their cyclic permutations. Then, for two octonion $x$ and $y$ given by

$$
\mathrm{x}=\sum_{\mu=0}^{7} \mathrm{x}_{\mu} \mathrm{e}_{\mu}, \quad \mathrm{y}=\underset{\substack{\sum_{\mu} \mathrm{y}_{\mu} \mathrm{e}_{\mu} \\ \mu=0}}{7}
$$

and for their product

$$
z=x y=\sum_{\mu=0}^{7} z_{\mu} e_{\mu}
$$

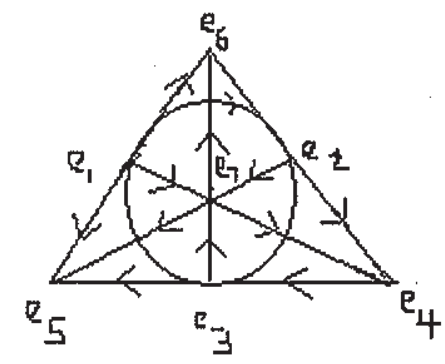


We obtain

$$
\underset{\mu=0}{\left.\sum_{\mu=0}^{7} x_{\mu^{2}}\right)^{2}} \underset{\delta=0}{\left(\sum_{\delta} y_{6}^{2}\right)}=\sum_{\mu=0}^{7} z_{\mu^{2}}
$$

we can also see that octonion algebra is non-associative. 8-dimensional algebra with basic $1, e_{0}, e_{1}, e_{2}, e_{3}, e_{4}, e_{5}, e_{6}, e_{7}$ and their multiplication is given in the following table which describe the result of multiplying the element in the ith row by the element in the jth column

\begin{tabular}{|l|l|l|l|l|l|l|l|}
\hline$\times$ & $e_{1}$ & $e_{2}$ & $e_{3}$ & $e_{4}$ & $e_{5}$ & $e_{6}$ & $e_{7}$ \\
\hline$e_{1}$ & -1 & $e_{4}$ & $e_{7}$ & $-e_{2}$ & $e_{6}$ & $-e_{5}$ & $-e_{3}$ \\
\hline$e_{2}$ & $-e_{4}$ & -1 & $e_{5}$ & $e_{1}$ & $-e_{3}$ & $e_{6}$ & $-e_{6}$ \\
\hline$e_{3}$ & $-e 7$ & $e_{1}$ & -1 & $e_{6}$ & $e_{2}$ & $-e_{4}$ & $e_{1}$ \\
\hline$e_{4}$ & $e_{2}$ & $-e_{1}$ & $-e_{7}$ & -1 & $e_{7}$ & $e_{3}$ & $-e_{5}$ \\
\hline$e_{5}$ & $-e_{6}$ & $e_{3}$ & $-e_{2}$ & $-e_{7}$ & -1 & $e_{1}$ & $e_{4}$ \\
\hline$e_{6}$ & $e_{5}$ & $-e_{7}$ & $e_{4}$ & $-e_{3}$ & $-e_{1}$ & -1 & $e_{2}$ \\
\hline$e_{7}$ & $e_{3}$ & $e_{6}$ & $-e_{1}$ & $e_{5}$ & $-e_{4}$ & $-e_{2}$ & -1 \\
\hline
\end{tabular}




\section{References}

1. Srinivasa Rao, K. N. "The Rotation and Lorentz Group and their representation for physicists".

2. Goldstein, H. "Classical Mechanics" second edition, reading, M. A. Addison-Welsey, P1511980.

3. Okcebo S. "Introduction to Octonions and other non associative algebras in physics".

4. Deavours C.A. "The Quaternion calculus". American mathematical monthly $1973 \mathrm{pp}$ 995-1008. 